
STAT-3 or DOCK-8

\title{
Síndrome hiper-lgE. Lecciones de la función y defectos de STAT-3 o DOCK-8
}

\author{
Julio César Alcántara-Montiel, ${ }^{1}$ Brittany Itzel Vega-Torres ${ }^{2}$
}

\begin{abstract}
In the classification of primary immunodeficiencies, hyper-IgE syndrome, identified with OMIM code \# 147060 in the Online Mendelian Inheritance in Man catalog, belongs to the group of syndromes associated with combined immunodeficiencies. It is characterized by elevated levels of IgE, eosinophilia, recurrent skin abscesses, pneumonia, lung parenchyma lesions, recurrent infections, rashes in newborns, eczema, sinusitis, otitis, and mucocutaneous candidiasis. Hyper-IgE syndrome can be transmitted by autosomal dominant or autosomal recessive modes of inheritance. Hyper-lgE syndrome in its dominant form includes non-immunological manifestations like characteristic facies, pathological dentition, scoliosis, bone disorders, and joint hyperextensibility. The reported cause of the dominant form is the loss of function of the signal transducer and activator of transcription 3 (STAT-3, with MIM \# 102582). Mutations in dedicator of cytokines 8 (DOCK-8) is the most common cause of the autosomal recessive form of hyper-IgE syndrome.
\end{abstract}

Keywords: Primary immunodeficiency; DOCK-8; Hyper-IgE syndrome; STAT-3

Este artículo debe citarse como: Alcántara-Montiel JC, Vega-Torres BI. Síndrome hiper-IgE. Lecciones de la función y defectos de STAT-3 o DOCK-8. Rev Alerg Mex. 2016;63(4):385-396

1 Universidad Nacional Autónoma de México, Facultad de Estudios Superiores Zaragoza, Facultad de Medicina. Ciudad de México, México

2Universidad Nacional Autónoma de México, Facultad de Estudios Superiores Zaragoza, Facultad de Química. Ciudad de México, México
Correspondencia: Julio César Alcántara-Montiel. jcalcantara@comunidad.unam.mx

Recibido: 2016-08-07

Aceptado: 2016-09-14 


\section{Resumen}

En la Clasificación de las Inmunodeficiencias Primarias, el síndrome hiper-lgE, identificado con el código OMIM \#147060 en el Catálogo Online Mendelian Inheritance in Man, pertenece al grupo de las inmunodeficiencias combinadas asociadas a síndromes. Se caracteriza por elevación de la concentración de IgE, eosinofilia, abscesos recurrentes en piel, neumonías, lesiones en parénquima pulmonar, infecciones recurrentes, erupciones en el recién nacido, eccema, sinusitis, otitis y candidiasis mucocutáneas. El síndrome hiper-IgE puede ser transmitido hereditariamente en forma autosómica dominante o autosómica recesiva. El síndrome hiper-IgE en su forma dominante incluye manifestaciones no inmunológicas como facies característica, dentición patológica, escoliosis, alteraciones óseas e hiperextensibilidad articular. La causa identificada en la forma dominante es la pérdida de la función del transductor de señales y activador de la transcripción 3 (STAT-3, MIM \#102582). Las mutaciones en la proteína dedicada a la citocinesis 8 (DOCK-8) representan la mayoría de las causas de la forma autosómica recesiva del síndrome hiper-lgE.

Palabras clave: Inmunodeficiencia primaria; DOCK-8; Síndrome hiper-lgE; STAT-3

\author{
Abreviaturas y siglas \\ CID, inmunodeficiencia combinada \\ GAP, proteínas activadoras de GTPasas \\ GEF, intercambiadores de nucleótidos de guanina \\ HIES, síndrome hiper-lgE
}

PBMC, células mononucleares de sangre periférica STAT-3, signal transducer and activator of transcription 3 VNC, variación del número de copias WAS, síndrome de Wiskott-Aldrich Y705, fosforilación de la tirosina 705

\section{Antecedentes}

Una inmunodeficiencia primaria en una perspectiva amplia es considerada como "cualquier anomalía del sistema inmune, la cual puede o no estar asociada con una enfermedad clínica". En la clasificación de las inmunodeficiencias primarias, el síndrome hiper-IgE (HIES), identificado con el código OMIM \#147060 en el Catálogo Online Mendelian Inheritance in Man, pertenece al grupo de las inmunodeficiencias combinadas asociadas a síndromes. ${ }^{2}$ La enfermedad también se conoce con el epónimo síndrome de Job, con base en la cita del Libro de Job, capítulo 2, versículo 7:

El adversario se alejó de la presencia del señor e hirió a Job con una úlcera maligna, desde la planta de los pies hasta la cabeza.

Davis, en su descripción original del HIES, describió a dos niñas pelirrojas, delgadas, quienes desarrollaban infecciones pulmonares, dermatitis e infecciones por Staphylococcus aureus con pérdida de los signos de inflamación (abscesos fríos). Anteriormente, para el diagnóstico solo se consideraba la tríada clínica de abscesos fríos recurrentes en piel por Staphylococcus aureus, neumonía e IgE elevada. ${ }^{3}$

La descripción de HIES incluye manifestaciones inmunológicas como elevación de la concentración de IgE, eosinofilia, abscesos recurrentes en piel, neumonías, lesiones en parénquima pulmonar, infecciones recurrentes, erupciones del recién nacido, eccema, sinusitis, otitis y candidiasis mucocutáneas. Entre las manifestaciones no inmunológicas están la retención de dientes primarios, escoliosis, fracturas de huesos con trauma mínimo, hiperextensión articular, facies característica en la adolescencia (frente prominente, aumento del diámetro intercantal, base de la nariz ancha, paladar alto), malformaciones congénitas en esqueleto y alteraciones vasculares. 
Las manifestaciones clínicas dependen de la edad, por ello en etapas tempranas el diagnóstico puede ser incierto. ${ }^{4-8}$

Las infecciones por Staphylococcus en HIES son recurrentes y por lo general se manifiestan durante los primeros años de vida con presentación fría, con lo que se enmascaran las manifestaciones cardinales de la inflamación (rubor, calor y dolor). Entre las bacterias más aisladas se encuentran Staphylococcus aureus, Streptococcus pneumoniae y Haemophilus influenzae. Las infecciones oportunistas pueden incluir Pneumocystis jiroveci, Cryptococcus sp. y Aspergillus fumigatus. ${ }^{9-11}$

El modo de herencia es autosómico dominante, con penetrancia completa y expresividad variable, como lo demostraron Renner y colaboradores ${ }^{12}$ al estudiar el patrón de herencia y la mutación en uno de los casos originales descritos por Davis en 1966.

Es importante mencionar que existen pacientes con síndrome hiper-IgE que muestran características clínicas más leves de la enfermedad asociadas con la presencia de un mosaico somático en el transductor de señales y activador de la transcripción 3 (STAT-3). ${ }^{13,14}$ Se han descrito mutaciones en todos los dominios de la proteína, pero se reconoce que el dominio de unión al DNA y SH2 es un "punto caliente" (donde ocurren mutaciones con mayor frecuencia). ${ }^{15}$ Las mutaciones constitutivas o de línea germinal presentan las manifestaciones más severas de la enfermedad. ${ }^{16}$

Recientemente se ha descrito que distintas mutaciones en el mismo aminoácido causan ganancia o pérdida de la función de STAT-3, mostrando la distribución de las mutaciones hipomórficas, con ganancia de función, asociadas con leucemias, linfomas y alteraciones autoinmunes. ${ }^{17}$ Por otra parte, se ha documentado que algunas mutaciones de línea germinal en STAT-3 son causa temprana de enfermedad autoinmune sistémica. ${ }^{18}$

\section{STAT-3 (signal transducer and activator of transcription 3 )}

Hasta ahora, la única proteína causante de la enfermedad en la forma autosómica dominante es el transductor de señales y activador de la transcripción tipo 3, código MIM \#102582, STAT-3 (signal transducer and activator of transcription 3). ${ }^{6,19}$ Esta proteína pertenece a la familia encargada de la transducción de señales y activación de la transcripción, la cual está presente desde anélidos hasta vertebrados. En los mamíferos se describen 7 genes de la familia STAT (1, 2 ,3 ,4, 5A, 5B, 6). La diversidad en la secuencia de aminoácidos (750 a 850) del dominio de activación y su amplia distribución permiten responder eficientemente a una gran variedad de estímulos extracelulares como citocinas y factores de crecimiento. ${ }^{20,21}$

Los primeros estudios de ligamiento genético relacionaban a HIES con el cromosoma $4,{ }^{22} \sin$ embargo, Choi y colaboradores, mediante hibridación in situ, encontraron que STAT-3 se localiza en $17 q 21.2 .^{23}$ El gen tiene 24 exones y codifica para una proteína de 770 aminoácidos, con un peso de 88 $\mathrm{KDa}$. Se describen tres isoformas:

- La isoforma 1, o canónica, de 770 aminoácidos.

- La isoforma 2, que pierde el aminoácido 701, pero conserva los aminoácidos 705 y 727 necesarios para la fosforilación y, con ello, la actividad de la proteína.

- La isoforma 3, o beta, que se caracteriza por tener un extremo carboxilo terminal más corto, pierde 45 aminoácidos conservando solo el 705 y pierde el 727, permitiendo la formación de dímeros, pero alterando la actividad transcripcional completa.

\section{Señalización de STAT-3}

STAT-3 tiene un papel relevante en:

- Las citocinas que utilizan la cadena gamma común (IL-2, IL-7, IL-9, IL-15, IL-21).

- Las citocinas que señalizan a través de gp130 (IL-6, IL-11, IL-27, IL-31, CNTF, CT-1, oncostatina $\mathrm{M}$, factor inhibidor de leucemia).

- La familia de IL-10 (IL-19, IL-20, IL-22, IL-24, IL-26).

- Los receptores de tirosina cinasa (EGF, FGF, GH, IGF1, M-CSF, PAF). ${ }^{4}$

Algunas manifestaciones cardinales de HIES se deben a la ausencia o alteraciones en la señalización de IL-6. Las proteínas de STAT-3 sin fosforilar se encuentran especialmente en el citoplasma en forma de monómeros. Sin embargo, pueden existir homodímeros de STAT-3 sin fosforilar, pero son poco estables y se disocian fácilmente. Evidencia actual muestra que las proteínas STAT se encuentran en el 
núcleo en forma de monómeros y homodímeros sin fosforilar en ausencia de estímulos. ${ }^{24}$

La vía de señalización requiere otras proteínas efectoras como la familia de las JAK, las cuales son muy conservadas. En mamíferos se describen 4 proteínas: JAK 1, 2, 3 y TYK2. Las proteínas JAK se unen al dominio intracelular de los receptores autofosforilándose y catalizando las reacciones de fosforilación en las proteínas de la familia STAT. Las proteínas STAT se activan rápidamente y son reclutadas a su receptor gracias a la interacción de su dominio SH2. La fosforilación de la tirosina 705 (Y705) en STAT-3 contribuye a la formación de los dímeros o heterodímeros con STAT-1. ${ }^{20,21}$ Esta vía de señalización, dependiendo del tejido, puede tener cierta plasticidad para activar preferencialmente algunos promotores en las células. La cinética de permanencia en el núcleo difiere entre la isoforma alfa, o canónica, y la beta, ya que esta última permanece por más tiempo dentro del núcleo. Lo anterior sugiere que la isoforma beta tiene una función reguladora dominante negativa. ${ }^{25} \mathrm{La}$ fosforilación constitutiva de STAT-3 beta presente en algunas líneas celulares permite la transcripción de algunos genes blanco con efecto contrario. Se ha indicado que la IL-5 es la responsable de inhibir la isoforma beta y su función dominante negativa. ${ }^{26}$

\section{Hiper-IgE autosómico recesivo (HIES-AR)}

En 2004 se describió una cohorte de 13 pacientes diagnosticados con síndrome hiper-IgE provenientes de familias consanguíneas, con un modo de herencia autosómico recesivo; algunas manifestaciones clínicas diferían de la forma dominante, entre las más relevantes se encontraba la alta susceptibilidad a infecciones virales cutáneas. ${ }^{27}$ En 2009 se identificó que la mayoría de los pacientes eran portadores de mutaciones bialélicas en la proteína dedicada a la citocinesis 8 (DOCK-8), MIM \#243700. ${ }^{27,28} \mathrm{El}$ síndrome hiper-IgE se manifiesta como una inmunodeficiencia combinada (CID) debido a alteraciones humorales y celulares, con una alta mortalidad en las dos primeras décadas de vida.

Con el empleo de microarreglos de alta resolución en pacientes diagnosticados con HIES-AR se encontraron pérdidas en el cromosoma 9 p24.3; en 2009 se describió por primera vez una asociación con la causa molecular. En cohortes más grandes se corroboró la ausencia del gen $D O C K-8$, desde un exón a prácticamente todo el gen. Posteriormente se observó que las mutaciones puntuales (codón de paro, alteraciones de corte y empalme e inserciones) provocan cambios en el marco de lectura, que llevaban a la pérdida de la función de la proteína de DOCK-8. En general, las mutaciones son homocigotas y en menor proporción, heterocigotas..$^{29-31}$

La deficiencia de DOCK- 8 causa el síndrome en humanos y ratones, con las siguientes manifestaciones: concentraciones elevadas de $\operatorname{IgE}$ en suero, eosinofilia, eccema, abscesos, infecciones persistentes cutáneas, de vías respiratorias altas y aparato gastrointestinal. Existe una alta prevalencia de alergias. ${ }^{31,32}$

La deficiencia de DOCK-8 provoca alta incidencia de infecciones cutáneas recurrentes, asma y alergias severas, problemas autoinmunes (vasculitis), predisposición a padecimientos hematológicos como linfoma de Burkitt y linfoma no Hodking. Algunas características fenotípicas no inmunológicas son complicaciones cerebrales no infecciosas como vasculopatía, encefalopatía, meningitis y abscesos.

Los agentes etiológicos más comunes de las infecciones en la deficiencia de DOCK-8 son cocos grampositivos, especialmente Staphylococcus aureus como agente principal de los abscesos cutáneos, el cual a pesar de su alta incidencia no es el agente principal de las infecciones de vías aéreas; los agentes de estas varían ampliamente, destacando los estreptococos del grupo A, neumococos, bacilos gramnegativos como Klebsiella, Haemophilus influenza y Escherichia coli, entre otros.

Los pacientes presentan infecciones recurrentes por herpes simple, eccema herpético, queratitis e infecciones anogenitales. Otros agentes virales son el Molluscum contagiosum, varicela zóster y el virus del papiloma, que produce verrugas planas y rugosas. Entre las infecciones fúngicas se observa principalmente la ocasionada por Candida albicans en piel y uñas, que puede afectar oído, boca y vagina. En menor proporción se observan las infecciones por especies de Aspergillus. . $^{2,31}$

Las mutaciones en el gen $D O C K-8$ representan la principal causa molecular de la forma recesiva. La primera proteína de la familia DOCK-180 se ha descrito como causa de algunas enfermedades en humanos. ${ }^{29}$ Es conocido que la variación del número de copias (VNC) en genes relacionados con el sistema inmune y genes cercanos a los centrómeros y te- 
lómeros varían ampliamente. Algunos reportes indican la existencia de un alto número de copias (264) en la región cromosómica, las cuales comprenden secuencias tipo Alu, elementos intercalados largos, repetidos terminales largos y repetidos en tándem, los cuales incrementan la posibilidad de generar pérdidas subteloméricas mediante una recombinación anormal de genes, o bien la actividad de los elementos transponibles con la consecuente interrupción de genes. ${ }^{29,30}$

\section{Dedicator of cytokinesis 8 (DOCK-8)}

El gen $D O C K-8$ se encuentra codificado en el cromosoma 9p24.3, gen de 46 a 48 exones dependiendo de la isoforma expresada. Es una proteína grande con una longitud aproximada de $250 \mathrm{~kb}, 2099$ aminoácidos y un peso molecular de $239 \mathrm{kDa} .{ }^{28} \mathrm{La}$ isoforma 2 carece de los aminoácidos 927-958 y tiene un peso molecular de $235 \mathrm{kDa}$. La isoforma 3 difiere de la secuencia canónica en la cual los aminoácidos 1-68 se encuentran ausentes y tiene un peso molecular de $230 \mathrm{kDa}$. Finalmente, la isoforma 4 carece de los aminoácidos 1-68 y 927-958 posee un peso molecular de $227 \mathrm{KDa}$. Actualmente no existen protocolos experimentales para identificar todas las isoformas, las cuales son generadas por corte y empalme alternativo. ${ }^{28}$

La familia de las proteínas Rho son un grupo de 22 moléculas que tienen la función de GTPasas de bajo peso molecular y son esenciales para la regulación del citoesqueleto de actina. Su activación se encuentra regulada por dos tipos de proteínas, los intercambiadores de nucleótidos de guanina (GEF) y las proteínas activadoras de GTPasas (GAP). ${ }^{28,33}$ La manera como las GEF regulan la forma celular ante un estímulo es modificando el estado activo e inactivo de las proteínas Rho; las GEF intercambian GDP por GTP de manera cíclica, activando las proteínas que posteriormente desencadenan modificaciones del citoesqueleto de actina. ${ }^{34}$

Las proteínas de la familia DOCK-180 constituyen una familia de intercambiadores recientemente descrita que incluye 11 proteínas relacionadas que contienen el dominio catalítico conocido como DHR-2, por lo que son conocidas como atípicas. La proteína DOCK-8 pertenece a la familia DOCK180. De acuerdo a nueva evidencia, esta familia está encargada de la mayor parte de la actividad enzimática como GEF, regulando diversos procesos celu- lares que involucran morfología celular, migración, división celular y polaridad, entre otros. ${ }^{33,35,36}$

DOCK-8 es una proteína que se encuentra ampliamente expresada a través de diversos tejidos: riñón, pulmones, páncreas y, en menor proporción, corazón, cerebro y músculo esquelético, entre otros; sin embargo, se expresa especialmente en células hematopoyéticas y células mononucleares de sangre periférica (PBMC), por lo cual no sorprende que un defecto en su función cause una inmunodeficiencia. Al realizar pruebas intracelulares se observa que DOCK-8 está presente a lo largo del citosol con un patrón granular, especialmente se observa en las extensiones celulares y en el núcleo, exceptuando el nucléolo.

En la cohorte de 64 pacientes con deficiencia de DOCK-8, informada por Engelhardt, se observó que $70 \%$ presentaba pérdidas del gen de unos cuantos pares de bases hasta pérdidas grandes y, en menor proporción, mutaciones puntuales (pérdida de sentido o de corte y empalme). ${ }^{31}$ Se ha observado que mutaciones en el dominio DHR-1 comprometen la actividad de las proteínas de la familia DOCK-180; la activación de la proteína no parece estar comprometida, sin embargo, la señalización sí, ya que segundos mensajeros como el fosfatidil inositol son incapaces de continuar la activación hacia las proteínas Rho. ${ }^{35}$ Mutaciones dentro del dominio DHR-2 afectan directamente la función catalítica de la proteína. Mutaciones puntuales en dominios diferentes a DHR-1 y DHR-2 alteran su estructura y función, probablemente alterando el reconocimiento y función de las proteínas con las que interactúa.

\section{Señalización de DOCK-8}

Esta familia de proteínas se encuentra conservada y tienen gran relevancia biológica, ya que la activación de Cdc42 y Rac está involucrada en diversos procesos como la quimiotaxis, migración de leucocitos a los nódulos linfáticos, invasión de células tumorales y migración de células epidermales y gonadales durante el crecimiento embrionario. DOCK-8 presenta 2 dominios conservados de alta homología a lo largo de todos los integrantes de su familia: DHR-2, que se encuentra próximo al carboxilo terminal, dominio en el cual reside su actividad catalítica; y DHR-1, ubicado hacia el dominio $\mathrm{N}$ terminal, el cual se une a lípidos membranales, lo que permite a las proteínas DOCK dirigir la señalización a lugares específicos 


\section{Autosómica dominante \\ Defectos en STAT-3 \\ Facies característica \\ Infecciones por Staphylococcus aureus, Streptococcus pneumoniae, Haemophilus influenzae}

Cuadro 1. Manifestaciones clínicas distintivas más frecuentes de las formas dominante y recesiva del sín drome hiper-lgE

- Alteraciones esqueléticas: escoliosis, fracturas sin trauma, retención de dientes primarios

- Anormalidades en la línea media: paladar hendido, lengua bífida, hemivértebras, otras anomalías vertebrales

Alteraciones de tejido conectivo (hiperlaxitud) Alteraciones vasculares: aneurismas coronarios y cerebrales.

Complicaciones pulmonares: bronquiectasias, neumatocele

Sin afectación del sistema nervioso central

Eccema moderado
Autosómica recesiva

Defectos en DOCK-8

Sin facies característica

- Infecciones virales: molusco contagioso, herpes, varicela, papiloma, verrugas

- Infecciones por bacterias intracelulares (Salmonella)

- Infecciones por micobaterias

Ausencia de alteraciones esqueléticas

Ausencia de alteraciones en tejido conectivo

Ausencia

Complicaciones pulmonares poco frecuentes

Afectación de sistema nervioso central

Eccema severo y de difícil control

Alergias ambientales muy frecuentes de la membrana plasmática mediante el reconocimiento del fosfatidil inositol trifosfato, principal producto de la fosfatidilinositol-3-cinasa, importante en los procesos de reorganización del citoesqueleto..$^{35,37}$

DOCK-8 es una proteína que cataliza el intercambio de GDP por GTP en proteínas Rho para su activación y posterior traducción de señales, ante estímulos celulares externos como citocinas, factores de crecimiento e interacción con otras células.

El dominio DHR-2 de DOCK-8 interactúa con las proteínas $\mathrm{Cdc} 42$ de manera dependiente, permitiendo la función de GTPasa, además de generar modificaciones conformacionales para la unión del dominio DHR-1 al fosfatidil inositol trifosfato que permite la interacción con proteínas adaptadoras que le confieren estabilidad, de acuerdo con lo observado en otras proteínas integrantes de la familia DOCK-180, como DOCK-1. ${ }^{35,36,38}$

La interacción de DOCK-8 con Cdc42 incrementa la formación de filopodios, modificando la polaridad celular, procesos de endocitosis, interacción célula-célula y regulando de manera importante la polimerización de actina y la activación de la proteína WASP en progresión del ciclo celular. ${ }^{33,39}$ En general, las GEF muestran interacción redundante, por ello la acción de Cdc42 puede verse disminuida pero no abolida en su totalidad en la deficiencia de DOCK $-8{ }^{28}$

La activación de las proteínas en las vías de señalización en las que DOCK-8 participa involucra la modificación del citoesqueleto de actina, una proteína ampliamente expresada en células eucariontes; es el principal componente del citoesqueleto que mediante la formación de andamios soporta y forma la célula. Su versatilidad permite el tráfico de vesículas, adhesión celular, motilidad celular y ciclo celular, entre otras. Al encontrarse altamente expresado en células hematopoyéticas, los defectos en DOCK-8 repercuten en la función de las células del sistema inmunológico.

Como puede suponerse, la proteína DOCK-8 y WASP tienen relación directa en ciertas vías de señalización, por ello el síndrome de Wiskott-Aldrich (WAS), OMIM \#30100, comparte algunas características de la deficiencia de DOCK-8 como eccema, IgE elevada, alergias, infecciones severas, susceptibilidad a autoinmunidad y cáncer. A pesar de sus semejanzas, clínicamente son fácil de diferenciar 


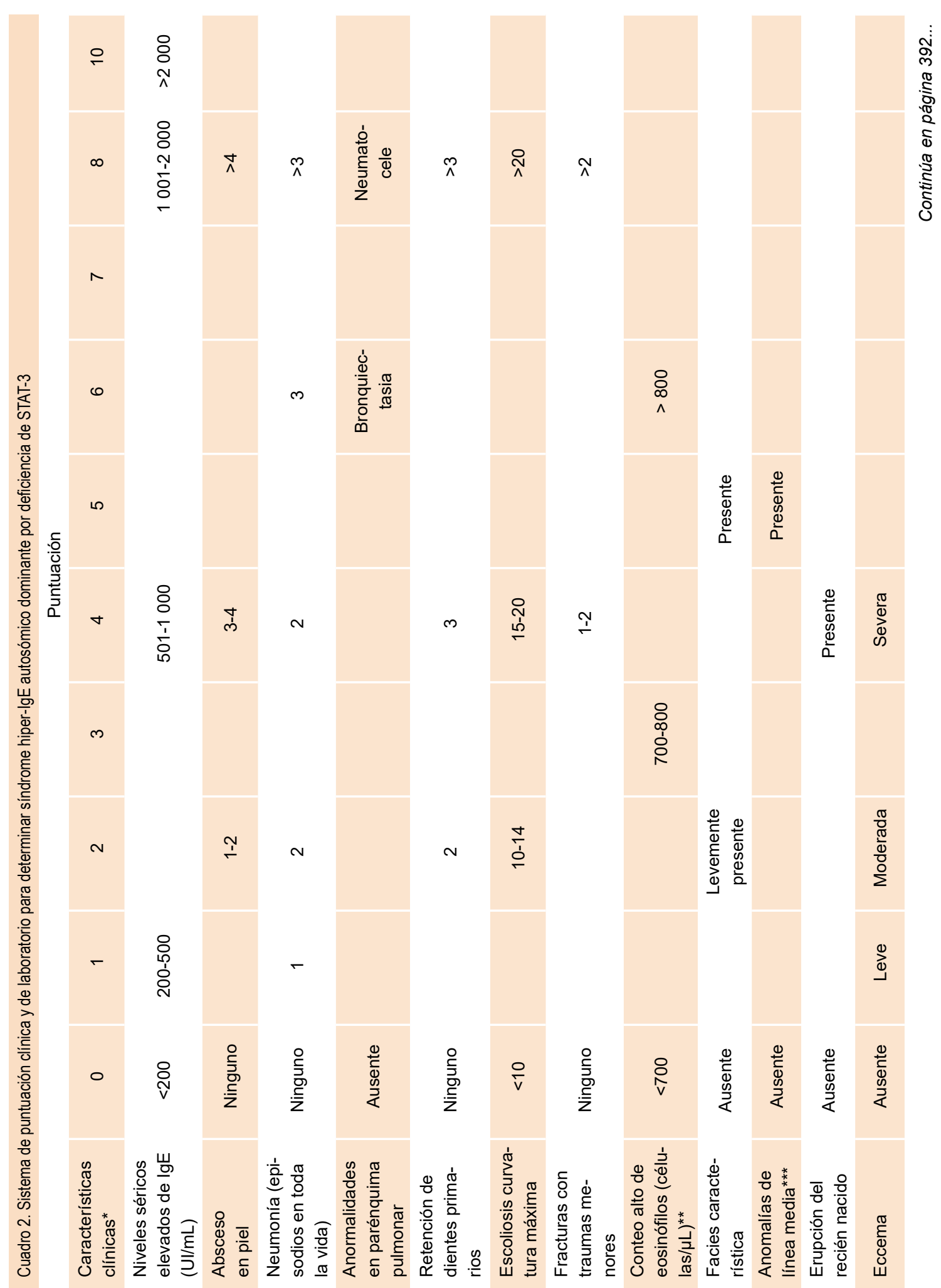




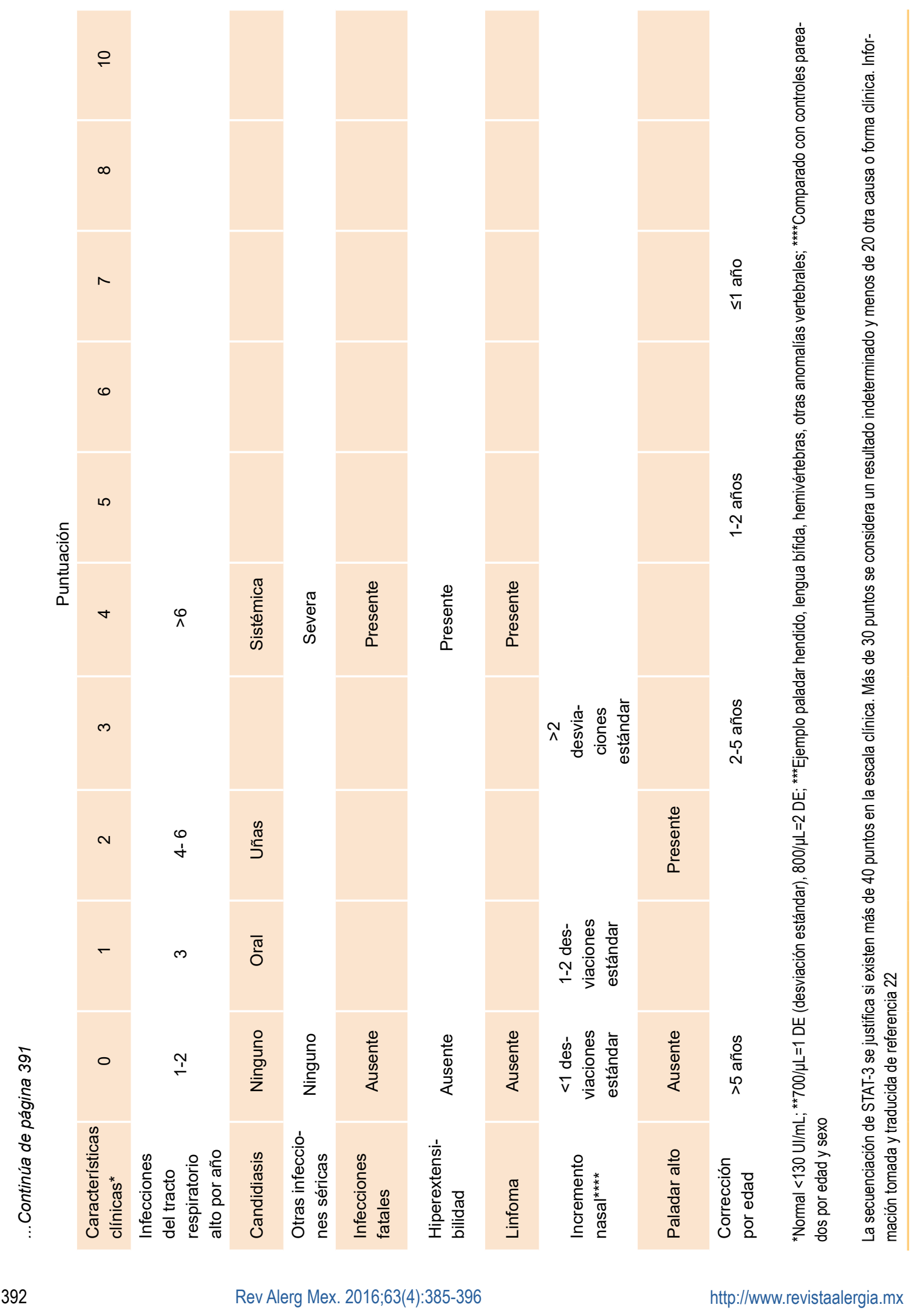


debido a que en WAS se presenta trombocitopenia y el modo de herencia está ligado al cromosoma X. ${ }^{28,32}$

Se han descrito de manera particular otras interacciones para DOCK-8 en células $\mathrm{B}$ y NK ; en el primer caso, DOCK-8 interacciona con las proteínas acopladora de TLR MyD88 y con la cinasa Pyk2; al unirse el ligando ADN no metilado CpG a TLR-9 este se activa, estabilizando la unión del complejo preexistente DOCK-8-MyD88-Pyk2, con ello Pyk2 fosforila a DOCK-8, lo cual propicia su interacción con otras cinasas como Src y Syk, que a su vez activan a STAT-3; esto podría establecer una conexión entre STAT-3 y DOCK-8, causantes moleculares principales de HIES-AD y HIES-AR, respectivamente. La regulación de ambas moléculas sobre la producción de células $\mathrm{B}$ de memoria de larga vida y secretoras de anticuerpos podría estar relacionada con el aumento de IgE en suero observado en el síndrome. ${ }^{40,41}$

Para las células NK se ha descrito que DOCK8 es importante para una citotoxicidad completa y la unión a su célula blanco. La interacción talinaDOCK-8 y WAS-DOCK-8 es muy importante para la regulación de los filamentos de actina y la maduración de las integrinas, las cuales se encuentran localizadas en el área de contacto celular en la sinapsis citotóxica de las células NK. Se observó que esta acumulación disminuye ampliamente en ausencia de DOCK $-8 .{ }^{42}$

\section{Diferencias entre las formas AD y AR}

Si se compara la deficiencia de DOCK-8 contra la de STAT-3 se puede distinguir clínicamente la forma recesiva de la enfermedad debido a la presencia de

\begin{tabular}{|c|c|c|c|c|c|}
\hline \multicolumn{2}{|c|}{ Necesario: lgE>10x mayor al rango } & \multirow{2}{*}{$\begin{array}{c}\text { Manifestación } \\
\text { Sin daños estruc- } \\
\text { turales }\end{array}$} & \multicolumn{2}{|c|}{ Puntos por escala } & \multirow{2}{*}{$\begin{array}{r}\text { Escala } \\
0.00\end{array}$} \\
\hline \multirow{3}{*}{ A } & \multirow{3}{*}{$\begin{array}{l}\text { Anormalidades del } \\
\text { parénquima pulmonar }\end{array}$} & & 0 & -5.00 & \\
\hline & & Bronquiectasias & 6 & -5.00 & -30.00 \\
\hline & & Neumatoceles & 8 & -5.00 & -40.00 \\
\hline \multirow{3}{*}{$\mathrm{B}$} & \multirow{3}{*}{ Eosinófilos altos $/ \mu \mathrm{L}$} & $<700$ & 0 & 8.18 & 0.00 \\
\hline & & $701-800$ & 3 & 8.18 & 24.54 \\
\hline & & $>800$ & 6 & 8.18 & 49.08 \\
\hline \multirow{4}{*}{$\mathrm{C}$} & \multirow{3}{*}{$\begin{array}{l}\text { Sinusitis, otitis (número } \\
\text { de episodios por año) }\end{array}$} & $1-2$ & 0 & 15.50 & 0.00 \\
\hline & & 3 & 1 & 15.50 & 15.50 \\
\hline & & $4-6$ & 2 & 15.50 & 31.00 \\
\hline & & $>6$ & 4 & 15.50 & 62.00 \\
\hline \multirow{5}{*}{$\mathrm{D}$} & \multirow{5}{*}{$\begin{array}{l}\text { Retención de dientes } \\
\text { primarios }\end{array}$} & Ninguno & 0 & -4.54 & 0.00 \\
\hline & & 1 & 1 & -4.54 & -4.54 \\
\hline & & 2 & 2 & -4.54 & -9.08 \\
\hline & & 3 & 4 & -4.54 & -18.16 \\
\hline & & $>3$ & 8 & -4.54 & -36.32 \\
\hline \multirow{3}{*}{$E$} & \multirow{3}{*}{$\begin{array}{l}\text { Fracturas con trauma } \\
\text { menor }\end{array}$} & 0 & 0 & -9.09 & 0.00 \\
\hline & & $1-2$ & 4 & -9.09 & -36.36 \\
\hline & & $>2$ & 8 & -9.09 & -72.72 \\
\hline \multicolumn{2}{|c|}{ Total de puntos en la escala (Sum A-E) } & & & & \\
\hline
\end{tabular}


anormalidades neurológicas, incremento en las infecciones virales de piel (Molluscum contagiosum, verrugas), ausencia de manifestaciones en tejido conectivo, alteraciones esqueléticas o retención de dientes primarios (Cuadro 1). A pesar de las diferencias fenotípicas entre los síndromes $\mathrm{AD}$ y $\mathrm{AR}$, en etapas tempranas y con síntomas comunes no es posible diferenciar certeramente la causa, la cual permitiría el tratamiento y asesoramiento adecuados para los pacientes. ${ }^{32,43}$ En los Cuadros 2 y 3 se describen las manifestaciones clínicas y de laboratorio para obtener las puntuaciones en las formas dominante y recesiva del síndrome
hiper-IgE por deficiencia de STAT-3 y DOCK-8. Un diagnóstico más certero ayudará a justificar la secuenciación para el diagnóstico molecular.

Finalmente, otras causas de la forma HIESAR incluyen los genes TYK2 y PGM3. Se han informado dos casos de mutación en TYK2 (OMIM \#243700). Recientemente se reportaron varias mutaciones en el gen de la enzima fosfoglucomutasa 3, PGM3 (OMIM \#615816). Los pacientes con fenotipo similar al síndrome hiper-IgE muestran atopia, elevación de $\operatorname{IgE}$, alteraciones neurológicas y del desarrollo. ${ }^{44,45}$

\section{Referencias}

1 Conley ME, Notarangelo LD, Casanova JL. Definition of primary immunodeficiency in 2011: A "trialogue" among friends. Ann N Y Acad Sci. 2011;1238:1-6. doi: 10.1111/j.1749-6632.2011.06212.x

2. Al-Herz W, Bousfiha A, Casanova JL, Chatila T, Conley ME, Cunningham-Rundles C, et al. Primary immunodeficiency diseases: An update on the classification from the international union of immunological societies expert committee for primary immunodeficiency. Front Immunol. 2014;5:162.

3. Davis SD, Schaller J, Wedgwood RJ. Job's syndrome. Recurrent, "cold", staphylococcal abscesses. Lancet. 1966;1(7445):1013-1015.

4. Minegishi Y. Hyper-IgE syndrome. Curr Opin Immunol. 2009;21(5):487-492. doi: 10.1016/j.coi.2009.07.013

5. Minegishi Y, Saito M. Cutaneous manifestations of hyper IgE syndrome. Allergol Int. 2012;61(2):191196. doi: 10.2332/allergolint.12-RAI-0423

6. Holland SM, DeLeo FR, Elloumi HZ, Hsu AP, Uzel G, Brodsky N, et al. STAT3 mutations in the hyper-lgE syndrome. N Engl J Med. 2007;357(16):1608-1619.

7. Freeman AF, Holland SM. Clinical manifestations, etiology, and pathogenesis of the hyper-lgE syndromes. Pediatr Res. 2009;65(5 Pt 2):32R-37R. doi: 10.1203/PDR.0b013e31819dc8c5

8. Freeman AF, Avila EM, Shaw PA, Davis J, Hsu AP, Welch P, et al. Coronary artery abnormalities in hyper-IgE syndrome. J Clin Immunol. 2011;31(3):338-345. doi: 10.1007/s10875-011-9515-9.

9. Heimall J, Freeman A, Holland SM. Pathogenesis of hyper IgE syndrome. Clin Rev Allergy Immunol. 2010;38(1):32-38. doi: 10.1007/s12016-009-8134-1

10. Freeman AF, Holland SM. Clinical manifestations of hyper IgE syndromes. Dis Markers. 2010;29(34):123-130. doi: 10.3233/DMA-2010-0734

11. Schimke LF, Sawalle-Belohradsky J, Roesler J, Wollenberg A, Rack A, Borte M, et al. Diagnostic approach to the hyper-IgE syndromes: Immunologic and clinical key findings to differentiate hyper-IgE syndromes from atopic dermatitis. J Allergy Clin Immunol. 2010;126(3):611-617.e1. doi: 10.1016/j.jaci.2010.06.029

12. Renner ED, Torgerson TR, Rylaarsdam S, Anover-Sombke S, Golob K, LaFlam T, et al. STAT3 mutation in the original patient with Job's syndrome. N Engl J Med. 2007;357(16):1667-1668.

13. Hsu AP, Sowerwine KJ, Lawrence MG, Davis J, Henderson CJ, Zarember KA, et al. Intermediate phenotypes in patients with autosomal dominant hyper-lgE syndrome caused by somatic mosaicism. J Allergy Clin Immunol. 2013;131(6):1586-1593. doi: 10.1016/j.jaci.2013.02.038

14. Alcántara-Montiel JC, Staines-Boone T, López-Herrera G, Ruiz LB, Borrego-Montoya CR, SantosArgumedo L. Somatic mosaicism in B cells of a patient with autosomal dominant hyper IgE syndrome. Eur J Immunol. 2016;46(10):2438-2443. doi: 10.1002/eji.201546275

15. Woellner C, Gertz EM, Schaffer AA, Lagos M, Perro M, Glocker EO, et al. Mutations in STAT3 and diagnostic guidelines for hyper-IgE syndrome. J Allergy Clin Immunol. 2010;125(2):424-432.e428. 
16. Freeman AF, Davis J, Hsu AP, Holland SM, Puck JM. Autosomal dominant hyper lgE syndrome. En: Pagon RA, Bird TD, Dolan CR, Stephens K, editores. GeneReviews. Seattle, WA: University of Washington; 1993

17. Chandrasekaran P, Zimmerman O, Paulson M, Sampaio EP, Freeman AF, Sowerwine KJ, et al. Distinct mutations at the same positions of STAT3 cause either loss or gain of function. J Allergy Clin Immunol. 2016;138(4):1222-1224.e2. doi: 10.1016/j.jaci.2016.05.007

18. Flanagan SE, Haapaniemi E, Russell MA, Caswell R, Lango Allen H, De Franco E, et al. Activating germline mutations in STAT3 cause early-onset multi-organ autoimmune disease. Nat Genet. 2014;46(8):812-814. doi: 10.1038/ng.304

19. Minegishi Y, Saito M, Tsuchiya S, Tsuge I, Takada H, Hara T, et al. Dominant-negative mutations in the DNA-binding domain of STAT3 cause hyper-IgE syndrome. Nature. 2007;448(7157):1058-1062.

20. Aaronson DS, Horvath CM. A road map for those who don't know JAK-STAT. Science. 2002;296(5573):1653-1655. doi: 10.1126/science.1071545

21. Levy DE, Loomis CA. STAT3 signaling and the hyper-IgE syndrome. N Engl J Med 2007;357(16):16551658. doi: 10.1056/NEJMe078197

22. Grimbacher B, Schaffer AA, Holland SM, Davis J, Gallin JI, Malech HL, et al. Genetic linkage of hyperIgE syndrome to chromosome 4. Am J Hum Genet. 1999;65(3):735-744. doi: 10.1086/302547

23. Choi JY, Li WL, Kouri RE, Yu J, Kao FT, Ruano G. Assignment of the acute phase response factor (APRF) gene to 17q21 by microdissection clone sequencing and fluorescence in situ hybridization of a P1 clone. Genomics. 1996;37(2):264-265.

24. Kretzschmar AK, Dinger MC, Henze C, Brocke-Heidrich K, Horn F. Analysis of Stat3 (signal transducer and activator of transcription 3) dimerization by fluorescence resonance energy transfer in living cells. Biochem J. 2004;377(Pt 2):289-297. doi: 10.1042/BJ20030708

25. Huang Y, Qiu J, Dong S, Redell MS, Poli V, Mancini MA, et al. Stat3 isoforms, alpha and beta, demonstrate distinct intracellular dynamics with prolonged nuclear retention of Stat3beta mapping to its unique C-terminal end. J Biol Chem. 2007;282(48):34958-34967.

26. Heinrich PC, Behrmann I, Muller-Newen G, Schaper F, Graeve L. Interleukin-6-type cytokine signalling through the gp130/Jak/STAT pathway. Biochem J. 1998;334(Pt 2):297-314.

27. Renner ED, Puck JM, Holland SM, Schmitt M, Weiss M, Frosch M, et al. Autosomal recessive hyperimmunoglobulin E syndrome: A distinct disease entity. J Pediatr. 2004;144(1):93-99.

28. McGhee SA, Chatila TA. DOCK8 immune deficiency as a model for primary cytoskeletal dysfunction. Dis Markers. 2010;29(3-4):151-156. doi: 10.3233/DMA-2010-0740

29. Zhang Q, Davis JC, Dove CG, Su HC. Genetic, clinical, and laboratory markers for DOCK8 immunodeficiency syndrome. Dis Markers. 2010;29(3-4):131-139. doi: 10.3233/DMA-2010-073

30. Engelhardt KR, McGhee S, Winkler S, Sassi A, Woellner C, López-Herrera G, et al. Large deletions and point mutations involving the dedicator of cytokinesis 8 (DOCK8) in the autosomal-recessive form of hyper-IgE syndrome. J Allergy Clin Immunol. 2009;124(6):1289-1302.e4. doi: 10.1016/j.jaci.2009.10.038

31. Engelhardt KR, Gertz ME, Keles S, Schaffer AA, Sigmund EC, Glocker C, et al. The extended clinical phenotype of 64 patients with dedicator of cytokinesis 8 deficiency. J Allergy Clin Immunol. 2015;136(2):402-412. doi: 10.1016/j.jaci.2014.12.1945

32. Aydin SE, Kilic SS, Aytekin C, Kumar A, Porras O, Kainulainen L, et al. DOCK8 deficiency: clinical and immunological phenotype and treatment options-a review of 136 patients. J Clin Immunol. 2015;35(2):189-198. doi: 10.1007/s10875-014-0126-0

33. Nishikimi A, Meller N, Uekawa N, Isobe K, Schwartz MA, Maruyama M. Zizimin2: a novel, DOCK180related Cdc42 guanine nucleotide exchange factor expressed predominantly in lymphocytes. FEBS Lett. 2005;579(5):1039-1046.

34. Nishikimi A, Kukimoto-Niino M, Yokoyama S, Fukui Y. Immune regulatory functions of DOCK family proteins in health and disease. Exp Cell Res. 2013;319(15):2343-2349. doi: 10.1016/j. yexcr.2013.07.024 
35. Cote JF, Vuori K. GEF what? Dock180 and related proteins help Rac to polarize cells in new ways. Trends Cell Biol. 2007;17(8):383-393.

36. Premkumar L, Bobkov AA, Patel M, Jaroszewski L, Bankston LA, Stec B, et al. Structural basis of membrane targeting by the Dock180 family of Rho family guanine exchange factors (Rho-GEFs). J Biol Chem. 2010;285(17):13211-13222. doi: 10.1074/jbc.M110.102517

37. Zhang Q, Dove CG, Hor JL, Murdock HM, Strauss-Albee DM, Garcia JA, et al. DOCK8 regulates lymphocyte shape integrity for skin antiviral immunity. J Exp Med. 2014;211(13):2549-2566. doi: 10.1084/ jem.20141307

38. Ruusala A, Aspenstrom P. Isolation and characterisation of DOCK8, a member of the DOCK180-related regulators of cell morphology. FEBS Lett. 2004;572(1-3):159-166.

39. Harada Y, Tanaka Y, Terasawa M, Pieczyk M, Habiro K, Katakai T, et al. DOCK8 is a Cdc42 activator critical for interstitial dendritic cell migration during immune responses. Blood 2012;119(19):4451-4461. doi: 10.1182/blood-2012-01-407098

40. Jabara HH, McDonald DR, Janssen E, Massaad MJ, Ramesh N, Borzutzky A, et al. DOCK8 functions as an adaptor that links TLR-MyD88 signaling to B cell activation. Nat Immunol. 2012;13(6):612-620. doi: 10.1038/ni.2305

41. Werner M, Jumaa H. DOCKing innate to adaptive signaling for persistent antibody production. Nat Immunol. 2012;13(6):525-526. doi:10.1038/ni.2317

42. Ham H, Guerrier S, Kim J, Schoon RA, Anderson EL, Hamann MJ, et al. Dedicator of cytokinesis 8 interacts with talin and Wiskott-Aldrich syndrome protein to regulate NK cell cytotoxicity. J Immunol. 2013;190(7):3661-3669. doi: 10.4049/jimmunol.1202792

43. Su HC. Dedicator of cytokinesis 8 (DOCK8) deficiency. Curr Opin Allergy Clin Immunol. 2010;10(6):515520. doi: 10.1097/ACl.0b013e32833fd718

44. Zhang Y, Yu X, Ichikawa M, Lyons JJ, Datta S, Lamborn IT, et al. Autosomal recessive phosphoglucomutase 3 (PGM3) mutations link glycosylation defects to atopy, immune deficiency, autoimmunity, and neurocognitive impairment. J Allergy Clin Immunol. 2014;133(5):1400-1409, 1409.e1-1409.e5. doi: 10.1016/j.jaci.2014.02.013

45. Sassi A, Lazaroski S, Wu G, Haslam SM, Fliegauf M, Mellouli F, et al. Hypomorphic homozygous mutations in phosphoglucomutase 3 (PGM3) impair immunity and increase serum IgE levels. J Allergy Clin Immunol. 2014;133(5):1410-1419, 1419 e1411-e1413. doi: 10.1016/j.jaci.2014.02.025 